\section{Bioethical Committees: Enablers or barriers of research?}

\section{Valeria Campos Cannobbio. ${ }^{1}$}

There's no discussion of the mandatory role of Bioethical Committees $(B C)$, as they provide an absolute respect to ethical principles in research, thus protecting the participants. Though BC are based on universal Ethical Laws, there's a lack of standardized criteria between Committees, not only around the world, but even inside the same institution (Cartes-Velasquez, 2017).

Generally, as most research projects are supported by a University, in order to conduct a research on-campus you need the University's BC approval. With the right guidance and patience, this step does not seem time-consuming. So, when do BC start becoming an obstacle for research?

When it comes to research off-campus, your institution's BC approval does not assure you anything, because you are going to need the approval of another BC, the BC from the public health service/School/Private practice where you want to conduct your research. Unfortunately, in some cases, they contradict the suggestions made by your institution's BC. So, here is the first dilemma. Does modifying the protocol recently approved by your academic institution, in order to get the other BC's approval, invalidates it? Should you inform your Institution's BC about any other changes suggested by the new BC?

Moreover, what happens when your research is not only off-campus, but multicenter? You need the approval from different BCs and each one of them will not only have different suggestions to get their approval, but also have a fee. Here is your second dilemma. Which suggestions should you follow? Should you have different informed consent forms according to each institution's BC you are conducting your research in? Is it ethical?

If this scenario isn't complex enough, in Chile, as most of public health centers depend on the Public Health Service, sometimes only in order to contact a public health center to conduct a research, they request the Public Health Service BC's approval. However, in order to obtain that approval, the BC needs a letter of endorsement from the public health center, which won't be provided without the Health Service BC's approval. Here is your third dilemma, as you get caught-up in a loop-hole. In addition to the above mentioned, in some cases BCs request you to deliver your research protocol in person, and that forces you or someone from your team to travel to different cities just to hand out a document.

In my experience, after my team and I have successfully solved this highly time-consuming obstacles in our multicenter research, I feel that neither of us wants to go again through this experience, and I dare to say that most researchers may be feeling the same way. In the future, this situation will negatively impact the improvement of health based on research, specifically in health care centers, as each time less and less researchers will feel that
INT J MED SURG SCI

Affiliations: ${ }^{1}$ Fundación Kimntrum, Concepción, Chile.

Corresponding author: Dra. Valeria Campos Cannobbio. Beltrán Mathieu 143-A. Phone: +56987690058. Email: vcamposc@ kimntrum.org.

Conflict of interests: None.

Acknowledgements: None.

doi: $10.32457 / \mathrm{ijmss} .2018 .033$. 
this whole process of different BCs' approvals is worth it (and necessary). As much as we want to promote evidencebased medicine, projects and grants have a due date, and any delay on this stage will reduce the time intended for data collection, data analysis and reports, transforming the fulfilling experience of research into a stressful one.

Finally, what can we do with these dilemmas? It is time to foster collaborative work between BCs, initiate dialogue, thus facilitating the progress of evidence-based medicine. In order to stop the CB's bureaucracy, here are some personal thoughts. Firstly, International experts on Ethical Laws should establish a Bioethical Guideline or Statement, such as STROBE, PRISMA, CONSORT (Tate \& Douglas, 2011), etc. to guide BCs around the globe to develop standardized criteria on the subject. Secondly, $B C s$ involved in the same research project should grasp from high-speed internet to network via videoconference in order to discuss the project and generate a unanimous suggestion. Thirdly, BCs must comply with reasonable due dates to deliver a decision on their reviews.

\section{REFERENCES}

Cartes-Velasquez RA. What is the role of the Bioethical Committees?. J Oral Res. 2017; 6(11), 278-279.

Tate RL, Douglas J. Use of reporting guidelines in scientific writing: PRISMA, CONSORT, STROBE, STARD and other resources. Brain Impair. 2011;12(1): 1-21. 